\title{
Study protocol of a randomized phase III trial of comparing preoperative chemoradiation with preoperative chemotherapy in patients with locally advanced gastric cancer or esophagogastric junction adenocarcinoma: PREACT
}

Xiaowen Liu ${ }^{1,2}$, Jiejie Jin ${ }^{1,2}$, Hong Cai ${ }^{1,2}$, Hua Huang ${ }^{1,2}$, Guangfa Zhao ${ }^{1,2}$, Ye Zhou ${ }^{1,2}$, Jianghong $\mathrm{Wu}^{1,2}$, Chunyan Du ${ }^{1,2}$, Ziwen Long ${ }^{1,2}$, Yantian Fang ${ }^{1,2}$, Mingze Ma ${ }^{1,2}$, Guichao Li $i^{2,3}$, Menglong Zhou ${ }^{2,3}$, Jiliang Yin ${ }^{2,4}$,

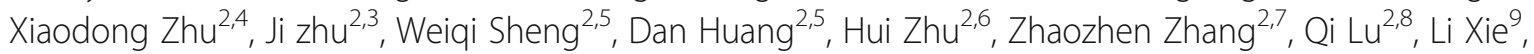
Zhen Zhang ${ }^{2,3^{*}}$ and Yanong Wang ${ }^{1,2^{*}}$

\begin{abstract}
Background: The prognosis of patients with locally advanced gastric cancer or esophagogastric junction adenocarcinoma is still dismal. There are no standard treatment strategies for these patients. Multidisciplinary team (MDT) approach is a good choice for making a high-quality decision. Generally, MDT will recommend these patients to receive preoperative chemotherapy or preoperative chemoradiation based on all kinds of treatment guidelines. However, the preferred preoperative treatment is still not established. In order to solve this problem, we carry out this randomized phase III trial of comparing preoperative chemoradiation with preoperative chemotherapy in patients with locally advanced gastric cancer or esophagogastric junction adenocarcinoma.

Methods: Eligible patients with locally advanced gastric cancer or esophagogastric junction adenocarcinoma are randomized to receive preoperative chemoradiation or preoperative chemotherapy, followed by surgery and postoperative chemotherapy. In the preoperative chemoradiation arm (Pre-CRT), patients receive two cycles of S-1 and oxaliplatin (SOX), chemoradiation, then followed by surgery and three more cycles of SOX chemotherapy. In the preoperative chemotherapy arm (Pre-CT), patients receive three cycles of SOX, following surgery three more cycles of SOX are given. The primary endpoint of this trial is to verify that preoperative chemoradiation could significantly improve the 3-year disease free survival (DFS) of patients with locally advanced gastric cancer or esophagogastric junction adenocarcinoma compared to preoperative chemotherapy.

Discussion: The results from this trial will provide important information about whether preoperative chemoradiation could improve survival compared to preoperative chemotherapy among patients with locally advanced gastric cancer or esophagogastric junction adenocarcinoma.

(Continued on next page)
\end{abstract}

\footnotetext{
*Correspondence: zhenzhang6@hotmail.com; wangyn1111@hotmail.com

2Department of Oncology, Shanghai Medical College, Fudan University,

Shanghai 200032, China

1 Department of Gastric Cancer Surgery, Fudan University Shanghai Cancer

Center, Shanghai 200032, China

Full list of author information is available at the end of the article
}

(c) The Author(s). 2019 Open Access This article is distributed under the terms of the Creative Commons Attribution 4.0 International License (http://creativecommons.org/licenses/by/4.0/), which permits unrestricted use, distribution, and reproduction in any medium, provided you give appropriate credit to the original author(s) and the source, provide a link to the Creative Commons license, and indicate if changes were made. The Creative Commons Public Domain Dedication waiver (http://creativecommons.org/publicdomain/zero/1.0/) applies to the data made available in this article, unless otherwise stated. 
(Continued from previous page)

Trial registration: ClinicalTrials.gov Identifier: NCT03013010. First posted January 6, 2017.

Keywords: Gastric cancer, Esophagogastric junction adenocarcinoma, Preoperative chemotherapy, Preoperative Chemoradiation, Gastrectomy, Adjuvant chemotherapy

\section{Background}

Gastric cancer is still the sixth most common cancer around the world, and approximately $40 \%$ of new patients with gastric cancer are diagnosed in China [1]. Surgery is still the most effective treatment modality. However, most Chinese patients with gastric cancer lose the chance to undergo surgery due to being diagnosed as advanced stage. Among these patients, those with locally advanced stage still have potential opportunity to receive surgery after preoperative chemotherapy or chemoradiation, which is also recommended by the NCCN guideline [2]. Based on MAGIC and FNCLCC/FFCD trial, perioperative chemotherapy has been established as an alternative option for patients with resectable gastric cancer $[3,4]$. Additionally, there are some preoperative chemoradiation reports in gastric cancer. A prospective, randomized trial showed that preoperative chemoradiation followed by surgery was superior to surgery in patients with resectable adenocarcinoma of the esophagus and gastric cardia [5]. Some phase II studies also showed that preoperative chemoradiation resulted in substantial pathologic response and $\mathrm{R} 0$ resection rate [6-8]. In our previous published research, $67 \%$ patients with locally advanced gastric cancer received curative gastrectomy after receiving chemoradiation [8]. Based on the publication of above-mentioned studies, clinicians have been faced with the dilemma of which preoperative therapy, chemotherapy or chemoradiation, should be applied to patients with locally advanced gastric cancer. A phase III study of comparing preoperative chemotherapy with chemoradiation was carried to solve this problem [9]. Unfortunately, this study was closed prematurely due to low accrual. Therefore, a randomized phase III trial comparing preoperative chemotherapy with chemoradiation is needed is deeply needed.

The aim of this study is to compare perioperative S-1 and oxaliplatin (SOX) chemotherapy with preoperative chemoradiation plus perioperative SOX chemotherapy in patients with locally advanced gastric cancer. Among the various chemotherapy regimes, $\mathrm{S}-1$ and oxaliplatin (SOX) appears to be effective. Some studies have shown that SOX had a favorable tumor response rate with a relatively mild toxicity profile $[10,11]$.The study protocol of this trial, which has the acronym PREACT, is described in this article. This trial is an prospective, multicenter study be conducted in China.

\section{Methods/design}

\section{Study setting}

PREACT is a randomized phase III trial, which is carried out at multicenter in China. Eligible patients with locally advanced gastric cancer or esophagogastric junction adenocarcinoma are randomized to receive preoperative chemoradiation or preoperative chemotherapy, followed by surgery and postoperative chemotherapy (Fig. 1). Patients were randomized by the way of stratified permutated block randomization on Web-based system. Lauren classification is stratification factor. In Pre-CRT arm, patients receive two cycles of SOX plus chemoradiation, followed by surgery and three more cycles of SOX chemotherapy. In the PreCT arm, patients receive three cycles of SOX, following

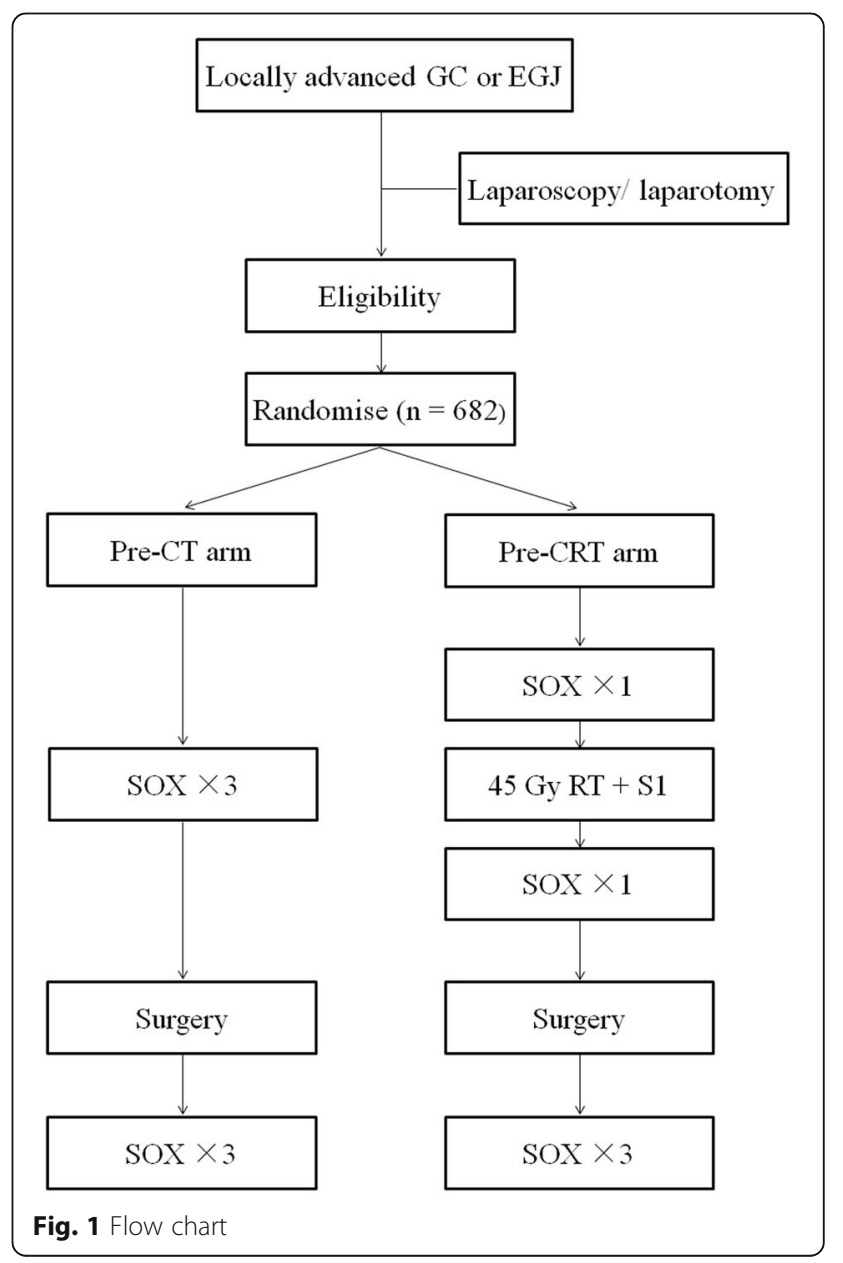


surgery three more cycles of SOX are given. The protocol has been approved by the Ethics Committee of Fudan University Shanghai Cancer Center, as well as other institutional ethics committees. All patients provided written informed consent before enrollment. Monitoring will be carried out in this trial.

\section{Primary endpoint}

3-year disease free survival (DFS).

\section{Secondary endpoint}

3-year overall survival (OS), curative gastrectomy rate, pathological response rate, treatment toxicity, and postoperative complications.

\section{Recruitment}

Patients with locally advanced, histologically confirmed gastric cancer or esophagogastric junction adenocarcinoma are recruited. All the patients should take following examinations: chest radiography, CT scan, and endoscopic ultrasonography. Six hundred and eighty-two patients will be enrolled in China. If the patients meets all the inclusion criteria, staging laparoscopy or laparotomy will be performed to exclude peritoneal dissemination.

\section{Inclusion criteria}

1) Age $\geq 18$ years.

2) Eastern Cooperative Oncology Group performance status $0-1$.

3) Life expectancy $\geq 6$ months.

4) Histologically proven adenocarcinoma of the stomach or esophagogastric junction(excluding Siewert I).

5) Clinical stage is stage IIB (T3 N1 only), IIIA (T2 N3 not eligible), IIIB, and IIIC, i.e. T3 -T4a and node positive, or T4b and/ or node positive, according to American Joint Committee on Cancer (AJCC) 7th edition.

6) Considered to be potentially resectable verified by a multi-disciplinary team including a surgical investigator.

7) Patients have adequate organ function as follows:

- Haemoglobin $\geq 90 \mathrm{~g} / \mathrm{L}$, Absolute neutrophil count $\left(\right.$ ANC) $\geq 1.5 \times 10^{9} / \mathrm{L}$, Platelet count $\geq 100 \times 10^{9} / \mathrm{L}$

- Serum bilirubin $\leq 1.5 \times$ upper limit of normal, Aspartate aminotransferase (AST) and/or alanine transaminase (ALT) $\leq 2.5 \times$ upper limit of normal

- Serum creatinine $\leq 1.0 \times$ upper limit of normal

8) Patients must provide the written informed consent.

\section{Exclusion criteria}

1) Pregnant or lactating females or planning to become pregnant or lactating.

2) There is evidence of metastatic disease.

3) Prior chemotherapy or radiotherapy.

4) Patients have a history of cancer in the five years before randomization except for the squamous or basal cell carcinoma of the skin that has been effectively treated, and carcinoma in situ of the cervix that has been treated by operation.

5) Patients with central nervous system (CNS) disorder or peripheral nervous system disorder or psychiatric disease.

6) Patients with known history of uncontrolled or symptomatic angina, uncontrolled arrhythmias and hypertension, or congestive heart failure, or cardiac infarction within 6 months prior to study enrollment, or cardiac insufficiency.

7) Patients with severe infection.

8) Patients with severe gastrointestinal bleeding, gastrointestinal perforation, or unable to swallow.

9) Patients with known hypersensitivity reaction or metabolic disorder to S-1or oxaliplatin in this study.

10) Patients with linitis plastica.

\section{Treatments}

\section{Chemotherapy}

SOX consists of S-1 and oxaliplatin. A course of chemotherapy will last 21 days. S-1 is administered orally with 40-60 mg, twice a day for 14 consecutive days, followed by a 7-day rest period (Fig. 2). The dose of S-1 was accorded to body-surface area (BSA): patients with a BSA of less than $1.25 \mathrm{~m}^{2}$ receive $80 \mathrm{mg}$ daily; those with a BSA of $1.25 \mathrm{~m}^{2}$ or more but less than $1.5 \mathrm{~m}^{2}$ receive $100 \mathrm{mg}$ daily; and those with a BSA of $1.5 \mathrm{~m}^{2}$ or more receive $120 \mathrm{mg}$ daily. Oxaliplatin $130 \mathrm{mg} / \mathrm{m}^{2}$, intravenously, on day 1.

\section{Chemoradiation}

Radiation is administered one week after completing the first cycle of chemotherapy. The treatment regimen consisted of $45 \mathrm{~Gy}$ of radiation delivered in 25 fractions ever five days per week for five weeks. Concurrent chemotherapy is $\mathrm{S}-140-60 \mathrm{mg} / \mathrm{m}^{2}$ orally, oral tablet twice daily, days $1-5$ of each week of radiotherapy (Fig. 3).Radiation treatment planning is done using computed tomography (CT) images. The radiation field includes the primary lesion and regional lymph node drainage.

\section{Surgery}

A standard D2 gastrectomy is recommended. The type of gastrectomy performed depends on the location and extent of the primary lesion. For middle third tumors, 


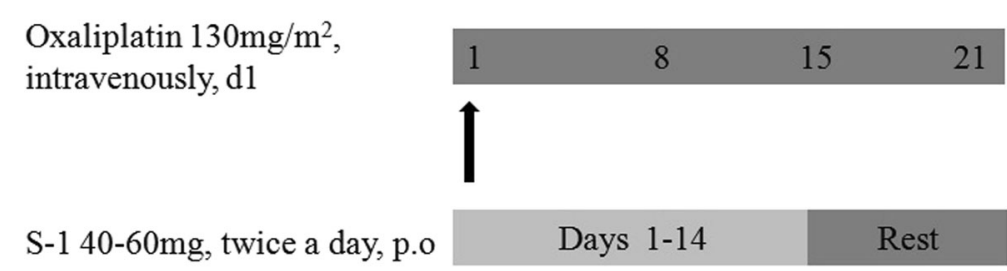

Fig. 2 Chemotherapy schedule

the gastric margin is recommended to be more than five $\mathrm{cm}$, and a total gastrectomy is performed. For lower third tumors, a two $\mathrm{cm}$ duodenal margin is recommended and a subtotal or total gastrectomy is considered. For upper third tumors, a three $\mathrm{cm}$ esophageal margin is recommended and a total gastrectomy or esophagogastrectomy is performed. Billroth I or Rouxen-Y gastrojejunostomy is performed for distal gastrectomy patients, Roux-en-Y esophagojejunostomy is performed for patients receiving total gastrectomy.

\section{Tumor response and toxicity criteria}

In the preoperative chemoradiation arm, tumor response evaluations are taken after chemoradiation and second circle of preoperative SOX by using abdominal CT scan. In the preoperative chemotherapy arm, abdominal CT scan was performed to evaluate tumor response after third cycle of preoperative SOX. All these evaluations are done according to the Response Evaluation Criteria for Solid Tumors (RECIST) 1.1. Adverse events were assessed according to Common Terminology Criteria for Adverse Event (CTCAE) v4.0.

\section{Follow up}

Follow-up of all patients was carried out according to our protocol (every three months for at least two years, every six months for years $3-5$, then every 12 months for life). Physical examination, tumor marker examination, and chest radiography were given every 3 months. Ultrasound and CT scan were given alternately every 6 months, and CT scan should be given at first follow-up. Endoscopic examination was given every 1 year [12].

\section{Sample size calculation}

In this study, patients with cT3N $+\mathrm{M} 0$, cT4aN $+\mathrm{M} 0$ or cT4bN- $/+\mathrm{M} 0$ are recruited. According to AJCC 7th edition, these patients belong to stage IIB, IIIA, IIIB, and IIIC. We found that the 3-year disease free survival (DFS) of same stage patients after D2 gastrectomy and adjuvant chemotherapy is $41 \%$ in our database. Based on CLASSIC study, which showed that patient with II or III could get a $14 \%$ increase of 3-year DFS in adjuvant chemotherapy arm comparing to alone surgery arm [13], we speculate that 3-year DFS will be $27 \%$ for such stage patients after receiving alone D2 gastrectomy. Based on MAGIC study, which showed that patient could get a 13\% increase of 3year DFS in perioperative chemotherapy arm comparing to alone surgery arm [3], we speculate that 3-year DFS will be $40 \%$ for such stage patients after receiving perioperative chemotherapy. This study assumes a $10 \%$ increase in 3year DFS favoring preoperative chemoradiation arm. Accrual time is four years followed by three years' follow up. Dropouts are set $10 \%$. It shows that a sample size of 682 will be needed with a $5 \%$ type I error (both sides) for a statistical power of $80 \%$.

\section{Statistical analysis}

DFS is calculated from the date of randomization to the date of detected disease recurrence. Following events are defined as recurrence: primary cancer recurrence, newly diagnosed gastric cancer, and death. OS is calculated from the date of randomization to the date of death or date of last follow-up. Survival is estimated by using the Kaplan-Meier method, and differences between survival curves are examined with log-rank test. Fisher's exact is used to compare patients' characteristics between preoperative chemotherapy arm and preoperative
Radiation $1.8 \mathrm{~Gy} \times 5$ days/week

S-1 40-60mg/m²/day, p.o, bid

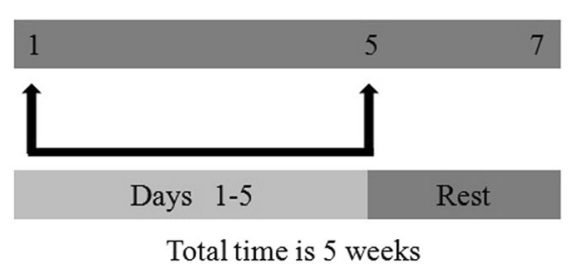

Total time is 5 weeks

Fig. 3 Chemoradiation schedule 
chemoradiation arm. Other secondary endpoints, including curative gastrectomy rate, pathological response rate, toxic effects, and postoperative complications, are compared between two arms using chi-square test. All statistical tests are two sides. The level of significance was $P<0.05$.

\section{Discussion}

This study is conducted to compare preoperative chemotherapy with preoperative chemoradiation in order to address which one is superior treatment in patients with locally advanced gastric cancer. Although this is not the first phase III trial of comparing preoperative chemotherapy with preoperative chemoradiation around the World, it has some advantages. As we all know, there are two obstacles needed to be addressed for preoperative therapy. First, most patients can not finish the scheduled treatment due to therapy toxicity. In this study, we use a combination of S-1 and oxaliplatin with concurrent radiation therapy as a preoperative treatment. SOX regimen has been proven to be effective and low-toxic, and has become a standard treatment in some Asian countries [14]. Therefore, we hypothesize that it may improve the patients' tolerance to chemotherapy. Second, clinical staging is not precise. Despite the fact that imaging techniques have substantially improved the diagnostic accuracy of infiltration and lymph node involvement, peritoneal dissemination can not be detected precisely. Especially, it is at high risk of intra-abdominal metastases in patients with locally advanced gastric cancer [15]. However, no clinical trials in gastric cancer mentioned how to solve this problem on the condition of preoperative chemotherapy or chemoradiation [3-7, $9,16]$. In this study, all the patients will receive exploratory laparotomy or laparoscopy and peritoneal cytological examination in order to exclude peritoneal carcinomatosis. Meanwhile, for patients with obstruction of gastric pylorus, we will perform by-pass surgery to improve the nutrition status.

In all, the results of this study will provide the first prospective multi-center data of comparing preoperative chemoradiation with chemotherapy in China, which will contribute to establish treatment standards for clinical practice in patients with locally advanced gastric cancer or esophagogastric junction adenocarcinoma.

\section{Abbreviations}

BSA: Body-surface area; CT: Computed tomography; DFS: Disease free survival; Pre-CRT: Preoperative chemoradiation; Pre-CT: Preoperative chemotherapy; SOX: S-1 and oxaliplatin

\section{Acknowledgements}

The authors thank the patients and the co-investigator for their cooperation in PREACT study.

\section{Funding}

This study was funded by grants from the Shanghai Committee of Science and Technology Funds (Contract grant numbers: 17411963200). The funders had no role in study design, data collection and analysis, decision to publish, or preparation of manuscript.

Availability of data and materials

The trial is ongoing and no data is available.

\section{Authors' contributions}

XWL, ZZ and YNW built the conception and designed the study. All authors contributed to the development of the study protocol. XWL, JJJ, HC, HH, GFZ, YZ, JHW, CYD, ZWL, GCL, JLY, XDZ, JZ, ZZZ, QL, MLZ, YTF, MZM and YNW were responsible for patients recruitment. WQS and $\mathrm{DH}$ were responsible for pathological examinations. $\mathrm{HZ}$ contributed to evaluate tumor response by $\mathrm{CT}$ scan. XWL, JZ and LX carried out the power calculation and data analysis. XWL wrote the first draft of the manuscript, and all authors commented and approved the final manuscript.

\section{Ethics approval and consent to participate}

This study is conducted in accordance with the "Declaration of Helsinki" and "Ethical Guidelines for Clinical Research", and the protocol has been approved by the Institutional Review Boards of each participating institute. Written informed consent is obtained from all patients before enrollment.

Institutional Review Board list:

Ethnic Committee of Fudan University Shanghai Cancer Center (Fudan

University Shanghai Cancer Center), committee's reference number:1611166-2. Huadong Hospital Ethnic Committee (Huadong Hospital), committee's reference number:2017 K052.

Ethnic Committee of Lishui Central Hospital (Lishui Central Hospital), committee's reference number:2018-56.

Shanghai Changhai Hospital Ethics Committee (Shanghai Changhai Hospital), committee's reference number:CHEC2018-075.

\section{Consent for publication}

Not applicable.

\section{Competing interests}

The authors declare that they have no competing interests.

\section{Publisher's Note}

Springer Nature remains neutral with regard to jurisdictional claims in published maps and institutional affiliations.

\section{Author details}

${ }^{1}$ Department of Gastric Cancer Surgery, Fudan University Shanghai Cancer Center, Shanghai 200032, China. ${ }^{2}$ Department of Oncology, Shanghai Medical College, Fudan University, Shanghai 200032, China. ${ }^{3}$ Department of Radiotherapy, Fudan University Shanghai Cancer Center, 270 Dong An Road, Shanghai 200032, China. ${ }^{4}$ Department of Medical Oncology, Fudan University Shanghai Cancer Center, Shanghai 200032, China. ${ }^{5}$ Department of Pathology, Fudan University Shanghai Cancer Center, Shanghai 200032, China.

${ }^{6}$ Department of Radiology, Fudan University Shanghai Cancer Center, Shanghai 200032, China. 'Department of Endoscopy, Fudan University Shanghai Cancer Center, Shanghai 200032, China. ${ }^{8}$ Department of General Surgery, Huadong Hospital Affiliated to Fudan University, Shanghai 200040, China. ${ }^{9}$ Clinical Research Center, Shanghai Jiao Tong University School of Medicine, Shanghai 200025, China.

Received: 20 March 2018 Accepted: 16 May 2019

Published online: 20 June 2019

\section{References}

1. Ferlay J, Soerjomataram I, Dikshit R, Eser S, Mathers C, Rebelo M, Parkin DM Forman D, Bray F. Cancer incidence and mortality worldwide: sources, methods and major patterns in GLOBOCAN 2012. Int J Cancer. 2014;136:E359-86.

2. National Comprehensive Cancer Network. NCCN Clinical Practice Guidelines in Oncology: Gastric Cancer, V.1.2016. Available at http://www.nccn.org/ professionals/ physician_gls/pdf/gastric.pdf. 
3. Cunningham D, Allum WH, Stenning SP, et al. Perioperative chemotherapy versus surgery alone for resectable gastroesophageal cancer. $N$ Engl J Med. 2006;355:11-20.

4. Ychou M, Boige V, Pignon J-P, et al. Perioperative chemotherapy compared with surgery alone for resectable gastroesophageal adenocarcinoma: an FNCLCC and FFCD multicenter phase III trial. J Clin Oncol. 2011;29:1715-21.

5. Walsh TN, Noonan N, Hollywood D, et al. A comparison of multimodal therapy and surgery for esophageal adenocarcinoma. N Engl J Med. 1996;335:462-7.

6. Ajani JA, Mansfield PF, Janjan N, et al. Multi-institutional trial of preoperative chemoradiotherapy in patients with potentially resectable gastric carcinoma. J Clin Oncol. 2004;22:2774-80.

7. Ajani JA, Mansfield PF, Crane CH, et al. Paclitaxel-based chemoradiotherapy in localized gastric carcinoma: degree of pathologic response and not clinical parameters dictated patient outcome. J Clin Oncol. 2005;23:1237-44.

8. Liu X, Li G, Long Z, Yin J, Zhu X, et al. Phase II trial of preoperative chemoradiation plus perioperative SOX chemotherapy in patients with locally advanced gastric cancer. J Surg Oncol. 2017. https://doi.org/10.1002/sso.24917.

9. Stahl M, Walz MK, Stuschke M, et al. Phase III comparing of preoperative chemotherapy with chemoradiotherapy in patients with locally advanced adenocarcinoma of the esophagogastric junction. J Clin Oncol. 2009;27:851-6.

10. Koizumi W, Takiuchi H, Yamada Y, et al. Phase II study of oxaliplatin plus S-1 as first-line treatment for advanced gastric cancer (G-SOX study). Ann Oncol. 2010;21(5):1001-5.

11. Oh SY, Kwon HC, Jeong SH, et al. A phase II study of S-1 and oxaliplatin (SOX) combination chemotherapy as a first-line therapy for patients with advanced gastric cancer. Investig New Drugs. 2012;30(1):350-6.

12. Liu $X, X u Y$, Long $Z$, Zhu H, Wang Y. Prognostic significance of tumor size in T3 gastric cancer. Ann Surg Oncol. 2009;16:1875-82.

13. Bang YJ, Kim YW, Yang HK, Chung HC, Park K, Lee KH, et al. Adjuvant capecitabine and oxaliplatin for gastric cancer after D2 gastrectomy (CLASS): a phase 3 open-label, randomised controlled trial. Lancet. 2012;28:315-21.

14. Yamada Y, Higuchi K, Nishikawa K, Gotoh M, Fuse N, Sugimoto N, Nishina T, et al. Phase III study comparing oxaliplatin plus S-1 with cisplatin plus S-1 in chemotherapy-naïve patients with advanced gastric cancer. Ann Oncol. 2015:26:141-8.

15. Jiang CG, Xu Y, Wang ZN, Sun Z, Liu FN, Yu M, Xu HM. Clinicopathological analysis and prognostic significance of peritoneal cytology in Chinese patients wit advanced gastric cancer. ANZ J Surg. 2011;81:608-13.

16. Leong T, Smithers BM, Michael M, Gebski V, Boussioutas A, Miller D, Simes J, et al. TOPGEAR: a randomised phase III trial of perioperative ECF chemotherapy versus preoperative chemoradiation plus perioperative ECF chemotherapy for resectable gastric cancer (an international, intergroup trial of the AGITG/TROG/EORTC/NCICCTG). BMC Cancer. 2015;15:532

Ready to submit your research? Choose BMC and benefit from:

- fast, convenient online submission

- thorough peer review by experienced researchers in your field

- rapid publication on acceptance

- support for research data, including large and complex data types

- gold Open Access which fosters wider collaboration and increased citations

- maximum visibility for your research: over $100 \mathrm{M}$ website views per year

At BMC, research is always in progress.

Learn more biomedcentral.com/submissions 